\title{
Approximation of Bessel Beams with Annular Arrays
}

\author{
Sverre Holm and G. Hossein Jamshidi
}

Department of Informatics, University of Oslo, P. O. Box 1080, N-0316 Oslo, Norway (sverre@ifi.uio.no)

\begin{abstract}
It is shown that the limited-diffraction Bessel beam of order zero can be generated by a transducer with equal-area division of elements and with a fixed prefocus, i.e. conventional transducers used in medical imaging equipment. The element division implies that the scaling parameter must be chosen to contain the first lobe of the Bessel function in the first element. In addition the prefocus must be such that the array is steerable to infinite depth. Examples of low- $(3.5 \mathrm{MHz})$ and high-frequency (20 $\mathrm{MHz}$ ) arrays are shown where the approximate Bessel beam compares favorably with a spherically focused beam with a fixed focus. The main advantages are a sharper nearfield and a more uniform beamwidth.
\end{abstract}

\section{INTRODUCTION}

The Bessel beam is characterized by a larger and more uniform depth of field than spherically focused beams. Such beams have been suggested as a way of solving the problem of getting good focusing far away from a fixed transmit focus in medical imaging. The purpose of this study is to determine the beam properties of a system where a transducer designed for dynamic focusing is used to generate an approximate Bessel beam. In this way a Bessel beam for transmission and a dynamically focused beam for reception can be generated by the same transducer.

The Bessel beam of order 0 is a member of a family of limited-diffraction solutions of the wave equation. For an infinite aperture, the sound pressure for a wave of frequency $\omega$, at the observation point $\vec{r}=(x, y, z)$, and at time $t$ is proportional to [2]:

$$
\begin{aligned}
U(\vec{r}, t) & =J_{0}(\alpha \rho) e^{j(\beta z-\omega t)} \\
\alpha^{2}+\beta^{2} & =k^{2} \quad k=\omega / c
\end{aligned}
$$

where $J_{0}$ is the Bessel function of order $0, \alpha \leq k$ is a real, positive scaling parameter, $\beta$ is a real parameter, $k$ is the wavenumber, and $\rho=\sqrt{x^{2}+y^{2}}$ is the distance from the center axis of the transducer. Note that $\alpha=0$ gives the plane wave solution.

The Bessel beam is characterized by a $-6 \mathrm{~dB}$ beamwidth of:

$$
\rho_{F W H M} \approx 3.04 / \alpha
$$

The distance between the first zeros of the beam is:

$$
\rho_{0-0} \approx 4.81 / \alpha
$$

The beam is characterized by an infinite depth of field and a relatively large first sidelobe of $-8 \mathrm{~dB}$.

When the aperture is finite, Durnin [1] showed from geometric arguments that the depth of field is:

$$
z_{\text {max }}=R \sqrt{\left(\frac{k}{\alpha}\right)^{2}-1}
$$

where $R$ is the transducer's radius.

$\mathrm{Lu}$ and Greenleaf [2] have shown that the Bessel beam can be well approximated by a flat, finite circular aperture when annular rings are made at the locations of the zeroes of the Bessel excitation function. They made a $2.5 \mathrm{MHz}, 50 \mathrm{~mm}$ diameter, 10 ring Bessel transducer according to this principle, and adjusted the amplitude on each ring to be equal to the peak of the respective Bessel lobe. They also demonstrated experimentally that limited-diffraction beams could be generated.

Due to the high sidelobe level, the Bessel beam of order 0 cannot be used for both the transmit and receive beams in an imaging system. Lu and Greenleaf have therefore proposed that a Bessel beam can be used on transmit in order to avoid the problem of transmit defocusing. The receive beam should be a conventional dynamically focused beam. The Bessel transducer could in principle be used on both transmit 
and receive, since dynamic focus could be applied by dynamically varying the delay on each ring with time, but it would be even better if one could use an annular array transducer of the type that is in common use.

The preferred transducer for use with dynamic focusing is the equal-area transducer. When each element has the same area, the division between elements $n$ and $n+1$ is found at:

$$
r_{n}=R \sqrt{\frac{n}{N}} \quad n \in[1, N]
$$

where $N$ is the number of elements. The annular array should be prefocused to a depth, $F$, in order to minimize the number of elements and the length of the delay lines. The advantages of the equal-area division is that the elements will defocus equally much as the range moves away from the prefocus (Fresnel condition), and that the elements will have the same electrical characteristics. The phase error over each element for a beam coming from infinite depth can be found from the geometry to be:

$$
\varphi_{\infty} \approx \frac{\pi}{N S} \quad S=\frac{F}{R^{2} / \lambda}
$$

where $S$ is the Fresnel number, i.e. the ratio of the fixed focus and the nearfield-to-farfield transition depth. To steer to infinite depth, this delay should be less than about $\pi / 2$.

This array is the starting point of our study: approximation of Bessel beams on equal-area, mechanically focused annular transducers. The equal-

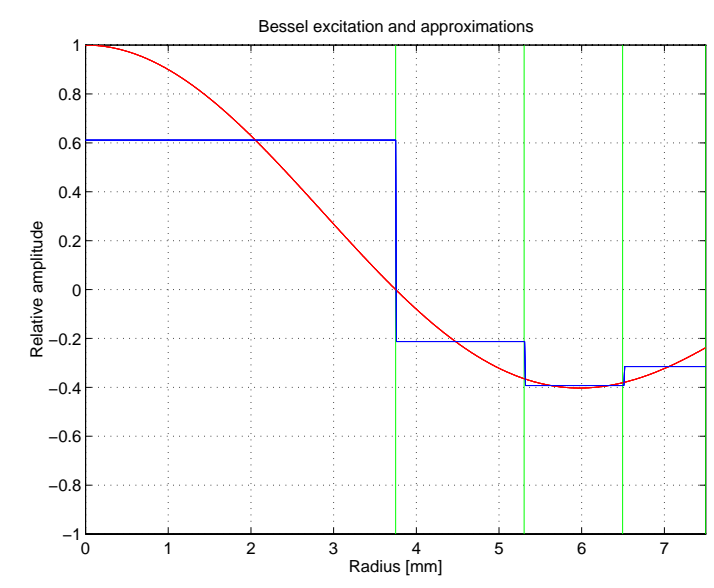

Fig. 1 Equal-area, exact Bessel excitation, and approximate excitation (piecewise constant curve). area assumption gives a relatively large radius for the inner ring, forcing the first zero of the Bessel excitation to be at this location. The scaling parameter $\alpha$ is therefore found by combining (3) and (5):

$$
\rho_{0-0}=2 r_{1}
$$

The solution is:

$$
\alpha=\frac{2.405 \sqrt{N}}{R}
$$

\section{LOW-FREQUENCY TRANSDUCER}

Fig. 1 shows the element division and excitation (envelope of (1) for $z=0$ ) for a $3.5 \mathrm{MHz}$ equal-area transducer with four rings and diameter $15 \mathrm{~mm}$. The scaling parameter from (8) is $\alpha=641.3 \mathrm{~m}^{-1}$.

The contour plot of the acoustic field for an ideally excited transducer, i.e. one where the excitation follows the continuous curve in Fig. 1, is shown in Fig. 2. Note the existence of the extra peaks from depth $175 \mathrm{~mm}$ and outwards as predicted by (4) for the depth-of-field $(167 \mathrm{~mm})$.

The effect of the step-wise approximation of the Bessel function, and the effect of the mechanical curvature must then be found. With a typical value for fixed focus of $F=75 \mathrm{~mm}$, the result is as shown in Fig. 3. The delays for each element has been set to compensate for the mechanical curvature. The Fresnel parameter is $S=0.59$ and the phase error for operation at infinity is $0.42 \pi$. Thus the effect of the

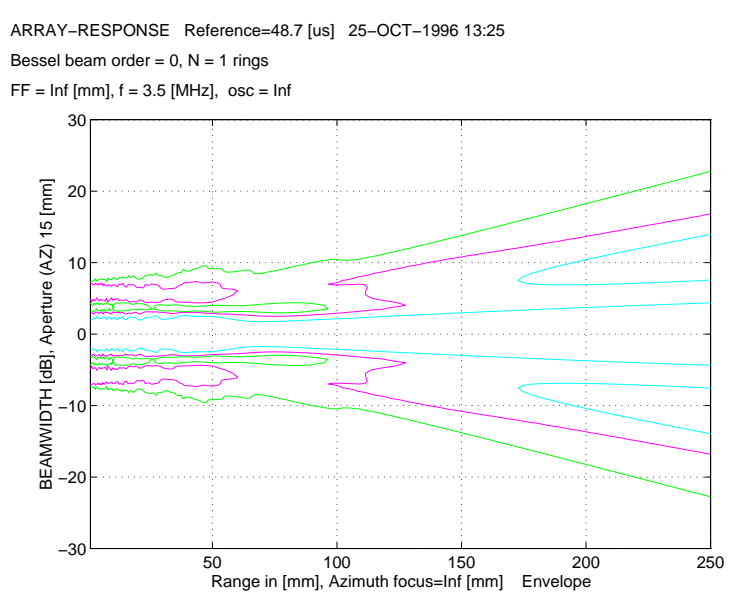

Fig. 2 Normalized contour plot showing -6, -12 , and $-18 \mathrm{~dB}$ contours for exact, finite aperture Bessel beam. 


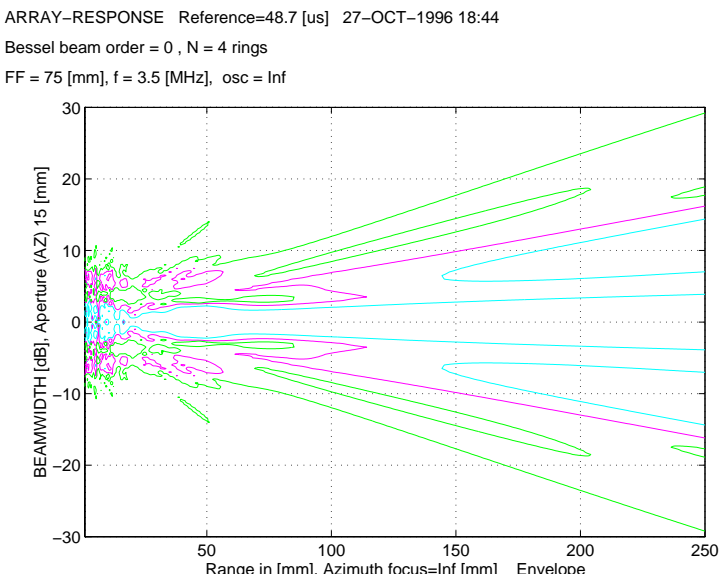

Fig. 3 Normalized contour plot showing -6, -12 , and $-18 \mathrm{~dB}$ contours for equalarea approximated Bessel beam with fixed focus.

curvature can be successfully compensated for by delays. There are some differences from Fig. 2, with the main one being an increased level of near-field artifacts and a reduction of the depth-of-field. Most of the deterioration comes from the step-wise approximation to the Bessel function.

The beam profile should be compared to the standard spherically focused transducer in Fig. 4. Further comparison can be made when the one-way profiles at specific depths are compared. The profiles at depths $12.5,25,75,125$, and $175 \mathrm{~mm}$ have been plotted with a $20 \mathrm{~dB}$ increment in Figs 5 and 6. The nearfield of the approximate Bessel beam is sharper,

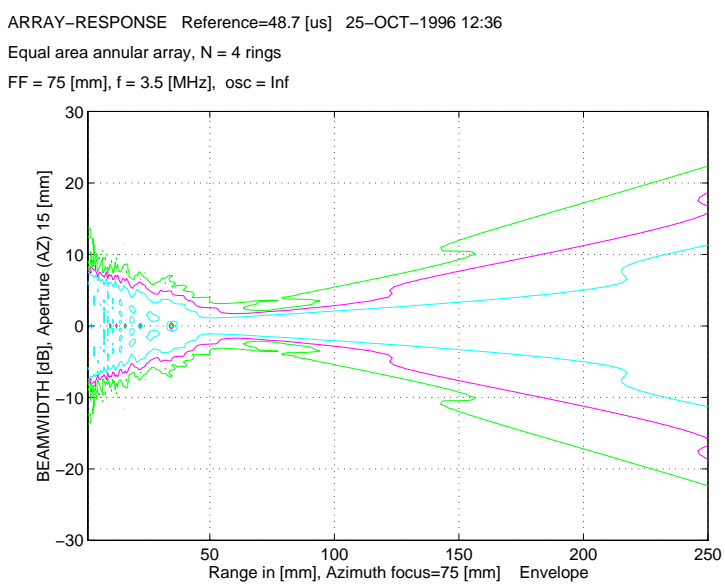

Fig. 4 Contour plot with -6, -12, and -18 dB contours for spherically focused transducer with fixed focus.

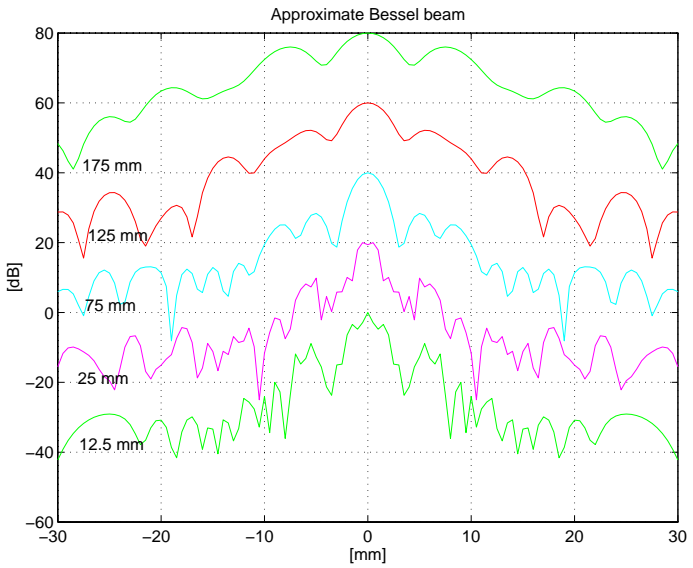

Fig. 5 One-way $\mathrm{CW}$ response at selected depths for Bessel beam (cuts through Fig. 3).

and there is a more uniform beamwidth. The spherically focused transducer has a sharper beam at its focus and it does not have the increase in sidelobes at the end of the depth-of-field.

A comparison of the on-axis intensities is shown in Fig. 7. Due to the absence of the sharp focusing around the fixed focus, the intensity distribution with depth is more uniform for the Bessel beam. This could be important in applications where peak intensities are limited.

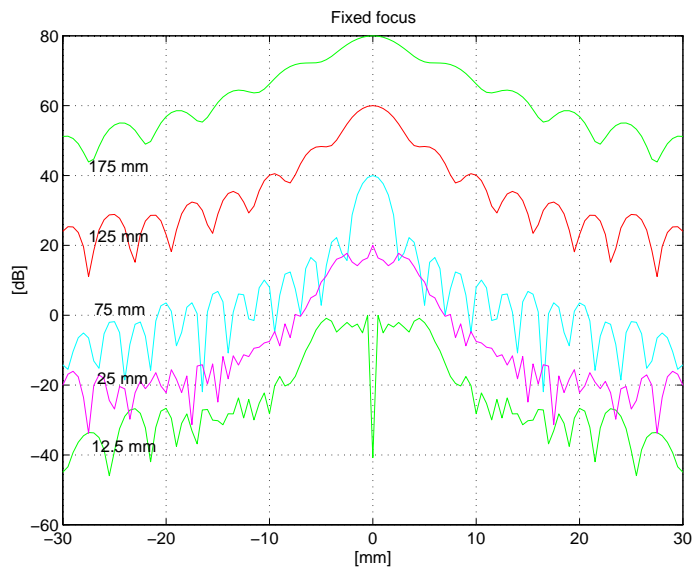

Fig. 6 One-way response at selected depths for beam with fixed focused at $75 \mathrm{~mm}$ (cuts through Fig. 4). 


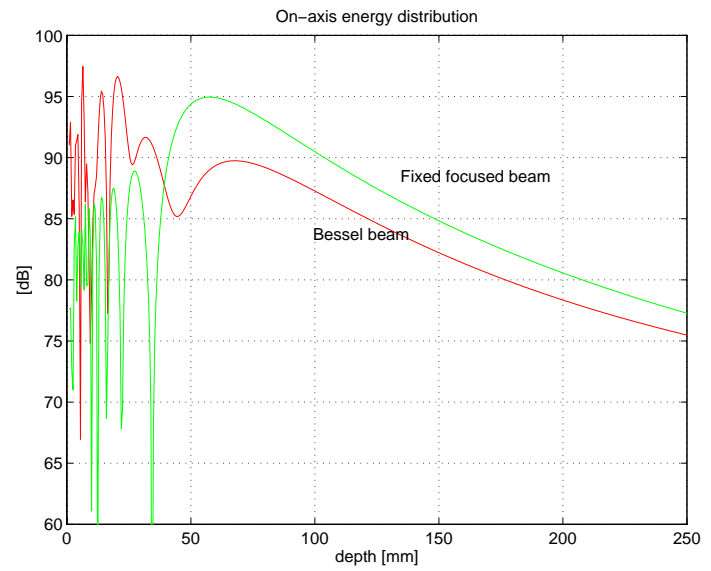

Fig. 7 On-axis intensities for approximate Bessel beam and for fixed focused beam $(75 \mathrm{~mm})$.

\section{HIGH-FREQUENCY TRANSDUCER}

This example is a $20 \mathrm{MHz} 1.4 \mathrm{~mm}$ transducer with $N=4$ rings. The approximate Bessel profile is shown in Fig. 8 and the fixed focus profile in Fig. 9. The excitation intensities are similar to the ones shown in Fig. 1, with the proper scaling for the difference in radius. The scaling parameter $\alpha$ is 6871 $\mathrm{m}^{-1}$ and the depth-of-field is predicted to $8.3 \mathrm{~mm}$. The characteristics of the beams for the Bessel and the spherically focused cases are similar to the ones for the low-frequency transducer.

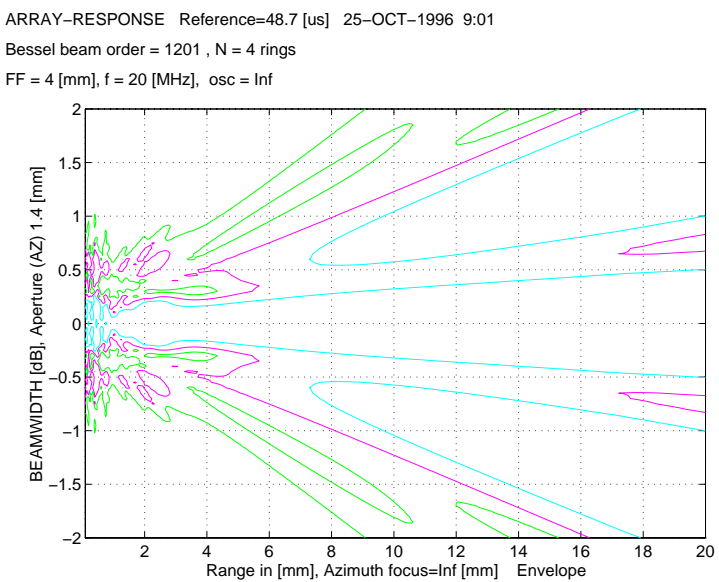

Fig. $8-6,-12$, and $-18 \mathrm{~dB}$ contours for approximate Bessel transducer, 1.4 mm at 20 MHz.

\section{CONCLUSION}

Both low- and high-frequency equal-area, prefocused annular arrays can be excited with approximate Bessel beams The scaling parameter of the Bessel beam of order 0 must be chosen to fit the first lobe of the Bessel function on the first element of the array, and the mechanical focus must be such that it can be compensated for by electronic delays. When these two conditions are met, good approximations to Bessel beams can be generated by equal-area, mechanically focused annular transducers. Further work is to determine the best approximation to the Bessel function on each element, e.g. the mean, the rms value, the peak or another approximation.

There is a trade-off between depth of field and beamwidth, but usually the Bessel beam will give better beams in the near- and farfields at the expense of a wider lateral beamwidth compared to a spherically focused beam.

\section{REFERENCES}

[1] J. Durnin, "Exact solutions for nondiffracting beams. I. The scalar theory," J. Opt. Soc. Am., Vol. 4, No. 4, pp 651-654, April 1987.

[2] J-Y. Lu and J.F. Greenleaf, "Ultrasonic nondiffracting transducer for medical imaging," IEEE Trans. Ultrason., Ferroelec., and Freq. Contr., Vol. 37, No. 5, pp. 438-447, Sept. 1990.

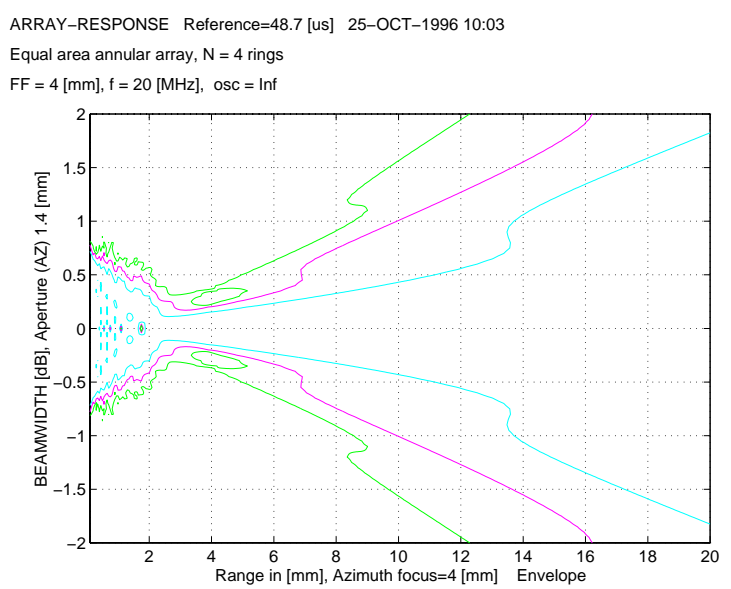

Fig. $9-6,-12$, and $-18 \mathrm{~dB}$ contours for spherically focused transducer, 1.4 $\mathrm{mm}$ at $20 \mathrm{MHz}$, fixed focus $4 \mathrm{~mm}$. 